\title{
Haptic Virtual Reality and Immersive Learning for Enhanced Organic Chemistry Instruction
}

Bosede Iyiade Edwards

Imagineering Institute, IDM Lab, Medini Mall, Nusajaya 79250, Johor;

City, University of London, Northampton Square, Clerkenwell, London EC1V 0HB, UK

bosede@imagineeringinstitute.org; +60187699318

Kevin S. Bielawski

Imagineering Institute, IDM Lab, Medini Mall, Nusajaya 79250, Johor;

City, University of London, Northampton Square, Clerkenwell, London EC1V 0HB, UK kevin@imagineeringinstitute.org

Rui F. Prada

Instituto Superior Técnico, Universidade de Lisboa, Portugal

rui.prada@tecnico.ulisboa.pt

Adrian David Cheok

Imagineering Institute, IDM Lab, Medini Mall, Nusajaya 79250, Johor

City, University of London, Northampton Square, Clerkenwell, London EC1V 0HB, UK

adrian@imagineeringinstitute.org 


\begin{abstract}
Human-Computer Interaction, including technology-aided instruction, is beginning to focus on virtual reality (VR) technology due to its ability to support immersive learning, teaching through simulation, and gamification of learning. These systems can deliver high-level multisensory learning experiences that are important in the teaching of many subjects, especially those involving abstract concepts or requiring spatial skills, such as organic chemistry. Haptic experiences with VR, however, remain a challenge. In addition, development have focused on general entertainment/gaming; VR systems in chemistry implement simulations of the chemistry laboratory and other advanced systems whereas those that support safe, game-like, immersive and multisensory learning of organic chemistry with haptics at pre-university education levels are scarce. We developed the VR Multisensory Classroom (VRMC) as an immersive learning environment within a VR head-mounted display, where learners employ hand movements to build hydrocarbon molecules and experience haptic feedback through gloves with built-in sensors and hand-tracking with the Leap Motion system. We report here the evaluation of the first prototype by learners from diverse backgrounds who reported on the ability of the VRMC to support high engagement, motivation, interest and organic chemistry learning as well as diverse learning styles. The VRMC is a novel VR classroom that supports immersive learning in molecular organic chemistry with haptics for multisensory learning.
\end{abstract}

\title{
KEYWORDS
}

Virtual Reality, Immersive Learning, Haptics, Chemistry Education, Organic Chemistry, Hydrocarbons, Middle School Science, Introductory Chemistry, Hands-On Learning, Gamification 


\section{INTRODUCTION}

Science, technology, engineering, and mathematics (STEM) education has become a key concept in basic education across the world, due to its pivotal nature to the learning of other subjects and its fundamental role in science and technological development of any nation. Increased funding, extensive research and development of learning technologies, including several multimedia systems, and pedagogical interventions in the delivery of STEM instruction continues with the principal aim of increasing performance in STEM subjects.

Multimedia learning systems have however focused largely on the visual and auditory senses to the exclusion of others like haptics and olfactory senses, thereby, reducing the additional advantages that focusing on these extra senses can deliver. Technology use in education had evolved over the years, and it is beginning to focus on Virtual Reality (VR) technology due to its ability to support immersive and collaborative learning (Christou, 2010; Monahan, McArdle, \& Bertolotto, 2008) and teaching through simulation (Hamid, Aziz, \& Azizi, 2014; Mujber, Szecsi, \& Hashmi, 2004), and gaming (Choi, Jung, \& Noh, 2015). VR is considered the next frontier of computer interaction (Fildes, 2015); it is employed in various types of training programmes (Farra, Miller, \& Hodgson, 2015; Yiannakopoulou, Nikiteas, Perrea, \& Tsigris, 2015), and it is specifically useful for supporting effective approaches to learner engagement and motivation (Buckley \& Doyle, 2016; Kuo \& Chuang, 2016). In fields like chemistry, where learners have to engage spatial skills (Dünser, Steinbügl, Kaufmann, \& Glück, 2006; Hauptman, 2010), VR can deliver outstanding advantages as educational technology and/or learning environment. In particular, it can support the visualization of abstract concepts like atoms, molecules, bonds, and others within an immersive environment.

Though several 3D applications exist for teaching chemistry, those that take advantage of haptics to improve multisensory learning are scarce, and those that combine haptics with 3D in an immersive environment are much rarer. Such systems can deliver great advantages in chemistry education especially in the learning of spatial orientations of organic molecules. The immersive environment can foster increased motivation, engagement and interest in chemistry as a subject (Mei \& Sheng, 2011), thereby increasing the interest of high school learners in STEM subjects. Leveraging VR with haptics for the teaching of chemistry can represent a novel approach to chemistry education; and a facilitating tool for tangible and multisensory instruction in a highly motivating learning environment. When properly designed, it can support more effective multimedia instruction and collaborative learning than non-immersive, or 3D systems offering physical tangibility.

We are interested in chemistry as a key STEM subject due to its place as a link to entry and performance in several fields, including engineering, medicine, pharmacy, bio-technology, and many others. More specifically, our focus is on organic chemistry instruction, and how novel technologies can be leveraged for addressing concepts which continue to constitute a challenge in the learning of structure and bonding in organic molecules. In this paper, we describe the VR multisensory Classroom (VRMC), a chemistry classroom with haptics in VR for the teaching of basic hydrocarbon bonding and molecule formation, considered as initial step in the understanding of more complex molecules. We also report findings from the initial evaluation of the system.

Our project focuses on the design and development of an immersive, haptic VR system for teaching basic hydrocarbon bonding and molecular structures in organic chemistry. Within the VRMC environment, learners can 'touch' atoms and bonds, view them in 3D space and bring them together to form molecules. They can also view the spatial distribution of molecules so formed, thereby experiencing the real-ness of concepts learnt in 2D classrooms. This can be extended to the learning of other chemistry concepts like structural and conformational isomerism as well as other relevant topics like periodicity, and atomic structures in inorganic chemistry. In this paper, we focus on a implementation and evaluation of the VRMC system. Though, for the design, we focus on basic organic chemistry, this represents only one of several uses that the system can be designed for; our report will therefore exclude issues of content, pedagogy, or learning strategies.

The remainder of this paper is divided into 6 sections: section 2 discusses related works and state-of-the-art in haptic VR for learning while section 3 presents a discussion of the underpinning theory and instructional systems design (ISD) for the VRMC system in addition to description of the general features of the system. In section 4, we report the initial evaluations of the VRMC, describing the procedure and methodology employed. We present findings from the evaluation in section 5 , and a brief discussion section in section 6 . Conclusions, implications of study and our future plans are outlined in section 7 . 


\section{STATE OF THE ART}

\subsection{Immersive learning with VR}

Immersiveness, is described as 'seeming to surround the audience, player...so that they feel completely involved in something' (Cambridge Dictionary, 2017) or 'providing, involving, or characterized by deep absorption or immersion in something (such as an activity or a real or artificial environment)' (Merriam Webster Dictionary, 2017). Immersion describes the experience of 'presence' or feelings of 'being there' usually associated with games (Sanders \& Cairns, 2010) and VR (Mestre \& Vercher, 2011; Tuveri, Macis, Sorrentino, Spano, \& Scateni, 2016). It implies a heightened sense of enjoyment (Sanders \& Cairns, 2010), that promotes engagement and the 'flow' experience (Nakamura \& Csikszentmihalyi, 2009; Whitson \& Consoli, 2009). Within VR environments, the user is completely removed from all other forms of visual engagement, and the focus on the virtual environment is almost total. The main theme of immersion is reality or its simulation of it, in manners that gets the user completely involved or absorbed. The significance for learning, in terms of learner engagement cannot be overemphasized. Immersion is therefore a major aim of instructional design and facilitation and should also be a key focus in pedagogy or educational technology selection. Though VR systems are increasingly available across various fields, the systems mostly focus on games and entertainment rather than as subject-focused educational technologies. The time is therefore ripe for more developmental efforts to focus on haptic VR systems that are dedicated to the teaching of various subjects.

\subsection{Haptic Multimedia and VR Systems}

Touch, or haptics is one of the vital ways we discover the world around us; it is a key non-verbal means of communication that can promote meaningful and active learning. However, haptics in learning is currently largely untapped (Minogue \& Jones, 2006). Progress in the development of multimedia systems has given birth to several 3D and VR systems; however, those that combine haptics with multimedia experiences are scarce. Virtual learning systems in chemistry have been reported in some studies. The virtual auto-titrator (Ritter \& Johnson, 1997) allows the user to manipulate auto-generated $\mathrm{pH}$ data in spreadsheets and obtain $\mathrm{pH}$ curves to determine titration endpoints on a graphical output. Winkelmann, Scott, and Wong (2014) reported on students' activity on an experiment in VR on Second Life; noting positive student perception that supports virtual laboratories as feasible alternatives to real ones. Stone (2007) also demonstrated the ability of virtual chemistry systems to support the reinforcement of the connections between classroom learning of chemistry principles and real-life applications, as well as to support self-paced independent learning using chromatography with virtual laboratory exercises. These systems do not employ haptics in their design, thereby failing to leverage the advantages of higher multisensory capabilities for improved learning. A major gap in general multimedia or VR technology in education thus remains those that combine haptics with immersive experience as the integration of haptics in VR remains a key challenge.

Currently available VR systems are mostly commercial applications and games, employing mostly hardware input. Axon VR (2017) is a haptic textile with feedback for texture, shape, vibration, etc.; it uses one hand for handling input hardware and the second hand for haptic experience. Hardlight suit and Haptx exoskeleton (Axon VR, 2017) are haptic feedback suits designed for gaming and entertainment. Dexmo, a hand haptic device for VR medical education by DextaRobotics (2018) is one of the few, existing, haptic VR learning systems. It uses force feedback to enable user feelings of size and shape, and captures 11 DoF of users' hand motion. Others, like GoTouch VR (2017) and Google VR (Google, 2017) are developer systems.

\subsection{VR and other Multimedia Systems for Organic Chemistry Instruction}

Development of VR systems for chemistry education has also grown and some of the systems currently available include MEL Chemistry VR (2017), Chemistry Experiment VR (MEL Chemistry, 2017), SuperChem VR (Schell Games, 2017), and Unimersiv Chemistry VR (2016). These systems focus mostly on simulation of experimental chemistry systems. 'Chairs!' (Winter, Wentzel, \& Ahluwalia, 2016), is a mobile application that teaches cyclohexane chemistry; it employs touchscreen as input. VR systems that focus on organic chemistry are scarce and limited in their capabilities; for example, in Stull, Barrett, and Hegarty (2013) a mouse interface is employed 
for manipulating virtual models. Chemistry VR systems applied as educational technologies and/or learning environments include MEL Chemistry (2017) which supports learning with a view of the molecular world but does not provide haptic experience. Chemistry Experiment VR (Merchant et al., 2013) is a VR-based chemistry laboratory for teaching high school experimental chemistry. It simulates haptic experience with 'triggers' using hardware. Superchem VR (Schell Games, 2017) is a similar system that uses HTC Vive for simulating haptics. Unimersiv Chemistry VR (Unimersiv, 2016) focuses on the periodic table and uses a cardboard magnet for simulating haptics. Devpost's Chemistry Lab VR (Smith, Agcaoili, \& Kannan, 2016) is a simulation of lab procedure with important safety instruction; it also uses hardware input through HTC Vive for haptics. Molecular Rift (Norrby, Grebner, Eriksson, \& Bostrom, 2015) simulates an advanced organic chemistry classroom with haptic VR, and it is designed for molecular visualization in drug design. It is the only one of the systems that employs direct hand manipulation rather than hardware. The need for an immersive VR system that employs direct hand manipulation in the teaching of basic organic chemistry thus becomes strong. A system that focuses on basic chemistry can help to address the challenge of beginner organic chemistry learners, thereby supporting the building of a strong foundation. We designed the VRMC, with a focus on teaching the nature and forms of bonding in hydrocarbon molecules.

\section{THE VRMC}

\subsection{Theoretical Framework and ISD}

Gamifying organic chemistry has been conceived by chemistry teachers as a tool to solve students' problems with learning the subject. Examples of chemistry game systems include 'Organic Molecule Game' (Woolley, Sheeley, \& Sheeley, 2010), 'Fastest Fingers' (Eastwood, 2013), 'React!' (React! Team, 2017), and 'Chairs!' (Winter, Wentzel, \& Ahluwalia, 2016). All these systems focus on the creation of game-like experiences which support learning through trial-and-error during play. This model is adopted in the design of the VRMC system. In addition, prior to the current extensive use of modern technologies in teaching and learning to simulate real-life application environments, it has been demonstrated that hands-on learning significantly affects attitudes toward science (Ornstein, 2006) and science achievement (Stohr-Hunt, 1996). The significance of hands-on or experiential learning has also informed several pedagogical approaches including service, problem-based and scenario-based learning, as well as science laboratory activities. The development of the VRMC is based on the significance and focus of experiential learning theory (ELT) as described by Kolb, Boyatzis, Richard, and Mainemelis, (2000), which addresses four concepts (Kolb \& Kolb, 2005). These are captured as abstract conceptualization (AC), that is, the generation of evidence from ideas and theories rather than from concrete examples and Concrete experience (CE), referring to being involved in a new experience. Reflective Observation (RO) is linked to personal experience or the experience of others as basis for developing observations while Active Experimentation (AE) involves using theories as a basis for problem-solving or decision-making.

The design of the VRMC is informed by these concepts, hence we developed an immersive learning environment where learners can build hydrocarbon molecules using hand motions, thereby taking advantage of learning through seeing, 'touching' (AC, CE), and bringing the abstract carbon and hydrogen 'atoms' together to form bonds (AC, RO), thus creating different types of hydrocarbon molecules (RO, AE). This experience of 'touching', 'taking', and 'making' plays significant roles in learning and describes the four elements (AC, CE, RO and AE) of ELT. Within the VRMC, abstract concepts like atoms, bonds, and molecules take 'concrete forms' and can be seen and touched due to the haptic sensory capability of the system. In this way, the generation of evidence becomes based on both theory and 'concrete examples' (AC), and the learners have novel experiences (CE) that forms the basis of problem-solving or decision-making (RO). In essence, the elements of ELT gets new meanings. The VRMC system is thus designed to support game-like approach to learning through building of organic molecules.

Approaches to ISD have been described in the literature (Heinich, Molenda, Russell, \& Smaldino, 2002; Jong, 2009; Molenda, 2003a). Most of the models follow a basic framework that identifies the five elements of analysis, design, development, implementation, and evaluation or ADDIE (Culatta, 2013; Molenda, 2003b). Table 1 highlights the development of VRMC in line with ADDIE model. 
Table 1. Instructional System Design for the VRMC based on ADDIE Model

\begin{tabular}{|l|l|}
\hline ID Element & Description in relation to VRMC \\
\hline Analysis & $\begin{array}{l}\text { The need to promote learner engagement, immersion, and learning that mimics real- } \\
\text { world multisensory stimulation; focus on STEM areas and promotion of learning of } \\
\text { abstract concepts and spatial skills in molecular organic chemistry }\end{array}$ \\
\hline Design & $\begin{array}{l}\text { VR-based learning environment that supports immersive learning for teaching of organic } \\
\text { chemistry; integration of haptics and gaming approach to learning }\end{array}$ \\
\hline Development & $\begin{array}{l}\text { VRMC with haptic input and support for multisensory instruction, immersive learning } \\
\text { and game design with atoms for building single, double or triple carbon-to-carbon bonds }\end{array}$ \\
\hline Evaluation & $\begin{array}{l}\text { System was deployed on VR gear with gloves to enable haptic feedback to users; natural } \\
\text { hand motions are employed in building hydrocarbon molecules with game-like } \\
\text { performance feedback to user as points scored for making bonds }\end{array}$ \\
$\begin{array}{l}\text { Based on the initial needs analysis, evaluation should focus on assessing the ability of the } \\
\text { system to deliver required solutions; for example, promoting learner engagement, } \\
\text { immersion, multisensory stimulation, haptics, tangible user-interfaces for learning, } \\
\text { support for learning of organic chemistry; support experiences beyond those of available } \\
\text { chemistry VR systems }\end{array}$ \\
\hline
\end{tabular}

\subsection{General Features of the VRMC}

VRMC integrates haptic input and supports multiple advantages for learning through tangible user-interfaces, multisensory instruction, immersive learning, and gamification. The initial system begins with only Carbon and Hydrogen as constituents of the simplest group of organic molecules, popularly known as hydrocarbons (HCs) which can exist as singly, doubly, or triply bonded molecules. We describe here, the VRMC interface and VR environment, the atoms, the bonding process, and the interaction and feedback in the system. We also provide a brief description of the basic modifications made in upgrading the initial system to the first tested version.

The VRMC system consists of an Android phone in a VR headset with a Leap Motion controller and haptic gloves. The system is controlled by a computer running Unity. A secondary display allows the observers to see a rendering of the VR environment. The carbon and hydrogen atoms shown as semi-transparent black and blue spheres, respectively. Different hydrocarbon molecules with single, double, and triple bonds can be made from the atoms and bonds. The user can also call for more atoms of carbon or hydrogen by 'pressing' the respective virtual buttons provided in the system. The VRMC environment with Ethyne $\left(\mathrm{C}_{3} \mathrm{H}_{3}\right)$, the first member of the triply bonded series, is shown in Figure 1.

The system implements three virtual buttons, as shown in Figure 1. The virtual button to the upper right side of the screen is used to check what molecule was made. It provides the user with feedback on whether a correct molecule was made or not and displays the name of the molecule. The virtual button to the upper left enables the user to add additional hydrogen atoms when needed for the creation of a molecule, while the virtual button to the lower right enables the user to add additional carbon atoms. A list of all atoms made by the user is shown at the bottom left corner of the screen. Feedback as points accruing to the user are based on bonds formed, each bond contributing two points to user scores, and is displayed at the top left of the environment. The system can be modified to include other types of atoms and to create different levels of task difficulty which can be made to correspond to varying levels of gaming experience. 


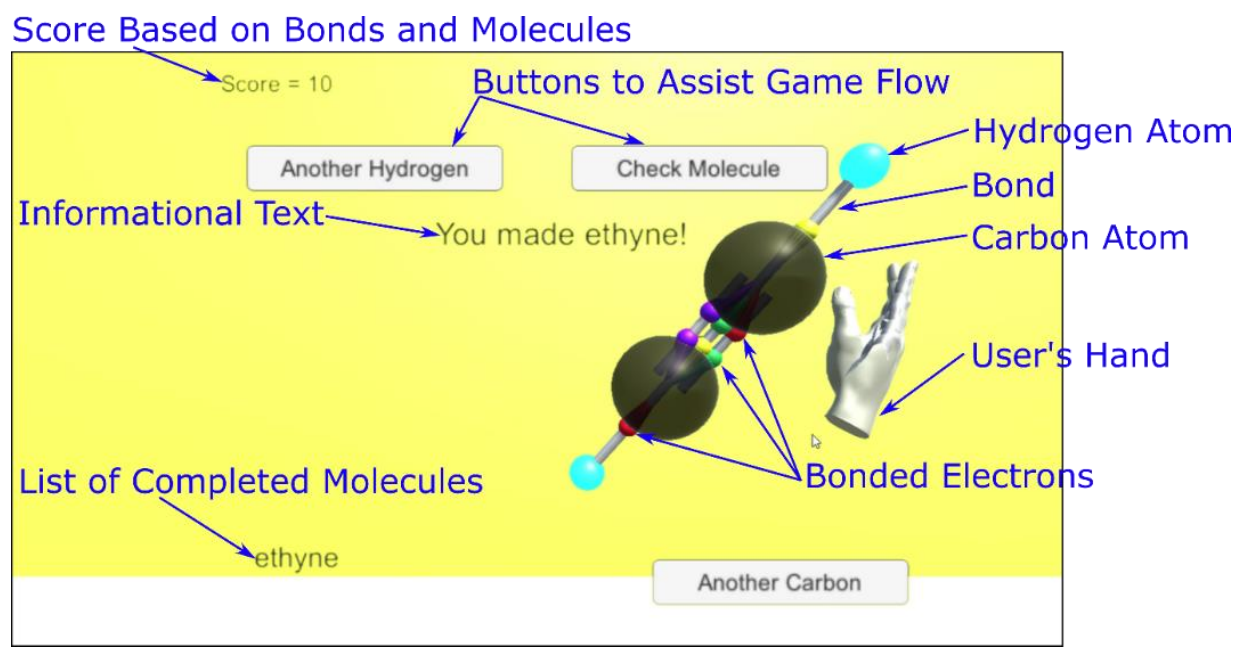

Figure 1. The VRMC with the triply-bonded ethyne has some features to control the game and guide the user in the creation of molecules.

Bonds between molecules are formed by grabbing an atom, bringing it into contact with another atom, then releasing it. Multiple bonds are formed by 'hitting' the atoms multiple times (twice for double bonds and thrice for triple bonds). The semi-transparent nature of the atoms makes it possible to view the spatial tetrahedral orientation of the $\mathrm{C}$ atoms at the actual 109.5 degrees. Electrons available for bonding are shown as coloured spots on the semi-transparent black carbon atoms. The spots are replaced by the bonds formed and become hidden after bonding. Visual and haptic feedback clues are provided to users to facilitate ease in building molecules. When the atom is ready to be moved, atoms change colour and the user experiences a haptic response in form of vibration so the user is given two forms of feedback that they can move the atom. While interacting with other atoms, the atom again changes colour with different haptic responses so the user can sense that a bond is ready to be made. The system comes with a pair of haptic gloves for providing input from and feedback to the user in conjunction with the Leap Motion system (Leap Motion, 2017) as shown in Figure 2.

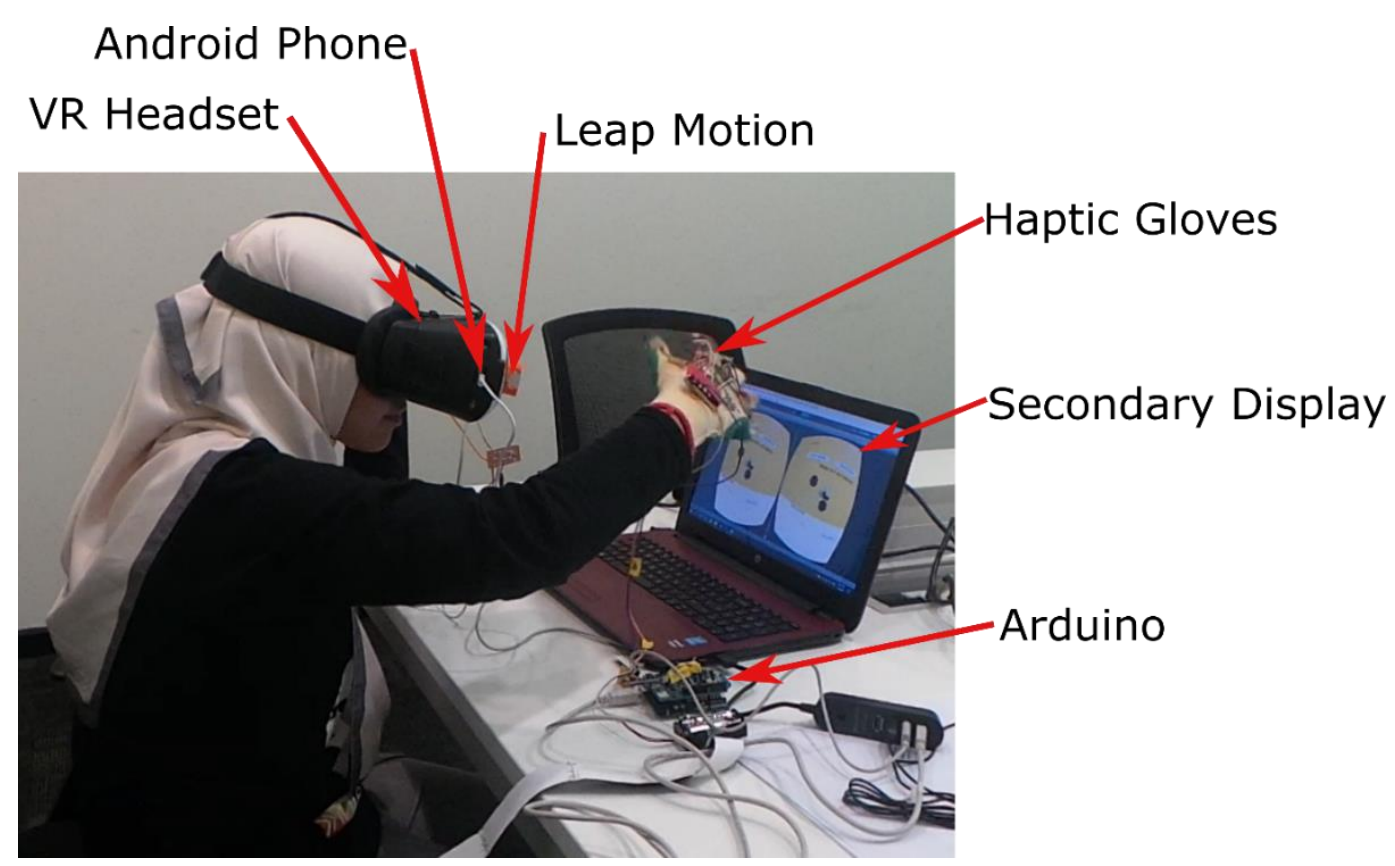

Figure 2: The VRMC system showing a user with different components of the system 


\subsection{Hardware implementation and User Interface Design}

A flex sensor continually measures if the user is grasping for an atom, and vibration motors at each fingertip provide feedback to the user. Signals for the flex sensor and vibration motors are controlled from an Arduino using a serial connection to the computer. The haptic glove consists of coin-type vibration motors at the finger tips, and a flex sensor along the middle finger. The motors and the sensor are connected to Arduino for control. The Arduino device communicates with the game through the serial port of a PC. The flex sensor enables precise monitoring of finger position, regardless of the orientation of the hand. This enhances the user experience, because the detection of grasping with Leap Motion can often create frustrating experiences. The haptic glove system with the flex sensor is shown in Figure 3.

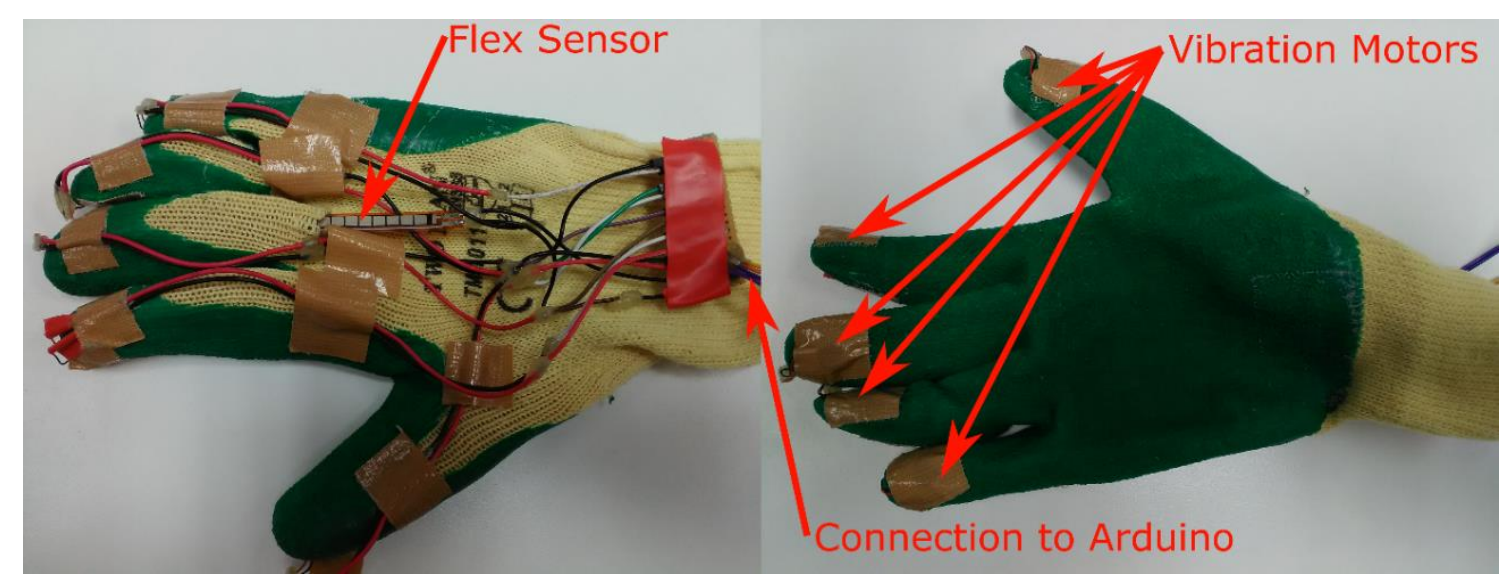

Figure 3. The haptic gloves provide feedback to the users through coin-type vibration motors, and the gloves contain a sensor to monitor the grasping of virtual atoms.

User-interface design provides opportunities for user-manipulation modelled after existing GUI systems, including laptops, mobile phones, and tablets, which feature either physical or touch-enabled 'buttons' to aid input. In designing the game-like virtual environment of VRMC, the system starts with minimal (2) number of atoms necessary for building a molecule, but with the possibility of the user getting more atoms if/when required. To achieve this, we implemented two buttons named 'Another Hydrogen' and 'Another Carbon'. User feedback is central in digital game design as well as in learning, hence, building of correct/incorrect molecule can be checked by users through the 'Check Molecule' button which is followed by relevant feedback from the system.

\section{EVALUATION OF THE VRMC SYSTEM}

User perceptions are central to determining future user experience and usability of various systems (Hyun, Yoon, $\&$ Son, 2010). The initial system evaluation was aimed at providing feedback on user perception, usability of the system and its adequacy for instructional purposes. Findings from the study were meant to answer the broad question: what are users' perceptions on the application of VRMC as an instructional tool for organic chemistry. The assessment is linked to the theoretical basis of VRMC's development, hence, we focus on assessment of the system's ability to support haptics (AC, CE), motivation/engagement (RO, AE), its ability to support collaborative learning and to serve as an instructional tool for organic chemistry, (CE, RO), and for the teaching of bonding through hands-on activities (AE, RO). We are also interested in the necessary improvement needs, based on userexperience.

The study engages a mixed design, that is, a combination of quantitative and qualitative approaches (Creswell, 2014), based on its ability to support richer feedback. Thus, a quantitative survey, and qualitative open-ended responses, and observations are employed in the study. Mixed approaches can employ any of sequential (qualitative after quantitative data collection or vice versa), nested (qualitative during quantitative data collection or vice versa), or concurrent (single-phase or quantitative and qualitative data collection in parallel) 
designs. We used the concurrent design, based on its ability to support complimentary results (Bazeley, 2010) as both types of data are given equal weights and are obtained within the same time frame (Creswell \& Plano Clark, 2007).

\subsection{Participants and Study Details}

Learners and teachers are the key stakeholders in any learning environment, and their feedback is central to the usability or effectiveness of any instructional system or technology. Our samples therefore consist of individuals across a wide age range but all participants assessed the VRMC from a learners' viewpoint. We sampled 13 users (7 females, 6 males), aged 12-36 years, including a chemistry teacher. This range of users can provide information on the utility and user experience with the VRMC as an instructional tool for individuals with different levels of familiarity with organic chemistry. Each participant wears the VR gear, and experiences the VRMC learning environment (LE). They were guided to explore the GUI and to carry out simple activities of building molecules using the bonds and the carbon and hydrogen atoms available within the LE.

\subsection{Briefing}

Participants were provided with an information sheet detailing the study procedure and requesting their informed consent before they participated in the study. The document provides game rules as the basics of hydrocarbon chemistry and how to build molecules, with an example shown for methane $\left(\mathrm{CH}_{4}\right)$. Participants were then expected to build molecules of their choice, ensuring that the conditions of bonding in carbon are fully satisfied in each case. Molecules built can be confirmed as (in)correct by 'pressing' a virtual button labelled 'check molecule' on the upper right section of the system. If there was more than one molecule in the system, the user will 'press' the 'check molecule' button and then touch the molecule to be checked. The system responds with statements like 'You have built methane!' Following the interaction session, the participants complete the short-survey.

\subsection{Instrumentation and Procedure}

Quantitative evaluation was based on a mini-survey while qualitative evaluations employed open-ended responses and participant observation during trial sessions with the system. We developed the mini-survey to capture the factors that are important regarding our system, as discussed above, hence, it assesses six characteristics, including (i) users' overall perception of the system, (ii) perception of haptics and experience of multisensory learning, (iii) experience of motivation, interest and engagement, and (iv) adequacy of the system for general and chemistry education. Participants rated each item as one of five options including 'very low', 'low', 'medium', 'high', and 'very high'. The internal consistency reliability of the mini-survey instrument based on Cronbach's alpha, $\alpha$, is 0.92 , this is a measure of 'scale reliability' or how closely related the set of items in the instrument are as a group. A high Cronbach's alpha value $(0.70-0.99)$ shows that an instrument is a reliable measure. The 'Cronbach's alpha if items deleted' values for all items of our mini-survey were between 0.91 and 0.92 , that is, similar to the Cronbach's alpha of the whole scale, and implying that item deletion will not improve the internal consistency of the instrument in any significant way. Hence, we used all the items in the instrument.

Participants' open-ended responses were designed to capture additional user comments and/or recommendations for system improvement. The protocol captured recommendations on seven characteristics, including general design, colour system, touch system, audio, instructional system, the haptic gloves and the design of the atoms. There was also a free-format feedback section for respondents to provide additional information on issues that might not have been captured in other sections.

Participants were immersed in the VR environment and were eliciting physical responses that are viewable only by observers; certain characteristics, like responses describing motivation, interest or enjoyment of the system, and physical feedback by users are only accessible through observation. Hence, the live observation was intended to achieve what Erlandson, Harris, Skipper, and Allen (1993) described as a "written photograph" of the situation being studied. An open (unstructured) protocol was designed to capture a multisensory description of the study in context. The observer records these descriptions as memo entries. 


\section{RESULTS}

\subsection{Quantitative Results}

The short survey instrument assessed participants' perceptions of the VRMC usability as an instructional tool based on its support for multisensory learning, haptics, motivation, and engagement. Its adequacy to serve as a learning tool both generally and specifically for chemistry as a subject were also assessed. The results of participants' evaluations are presented in Figures 4-9. Participants rating from "Very high" to Very Low" are plotted against the frequency of ratings which are the total number of times a construct is rated out of 52 possible times (13 persons on each of 4 items per construct). Ratings for each of the sub-constructs of multisensory learning, motivation and engagement, and haptics are plotted against frequencies. Participants' rating of the usefulness of system as an instructional tool, and specifically for chemistry instruction are also plotted in addition to participants' overall rating of the system. Figure 4 shows that the system is highly rated in terms of support for multisensory learning. There were 45 high/very high ratings out of a total of 52 with 7 average ratings and no "low" or "very low" ratings, indicating that on the whole, participants have very positive perception of the system in terms of its ability to support multisensory instruction.

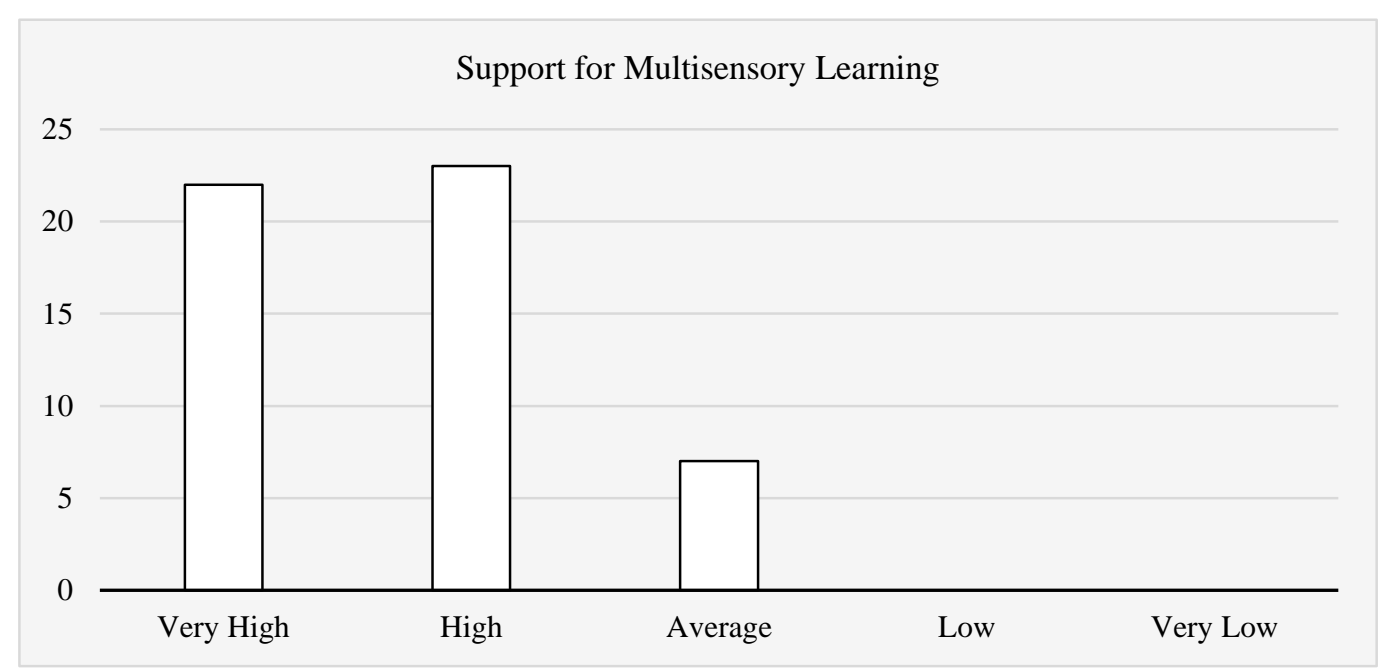

Figure 4: Participants’ Rating of System’s Support for Multisensory Learning

Rating of system's support for motivation and engagement are similar to that of multisensory learning as shown in Figure 5.

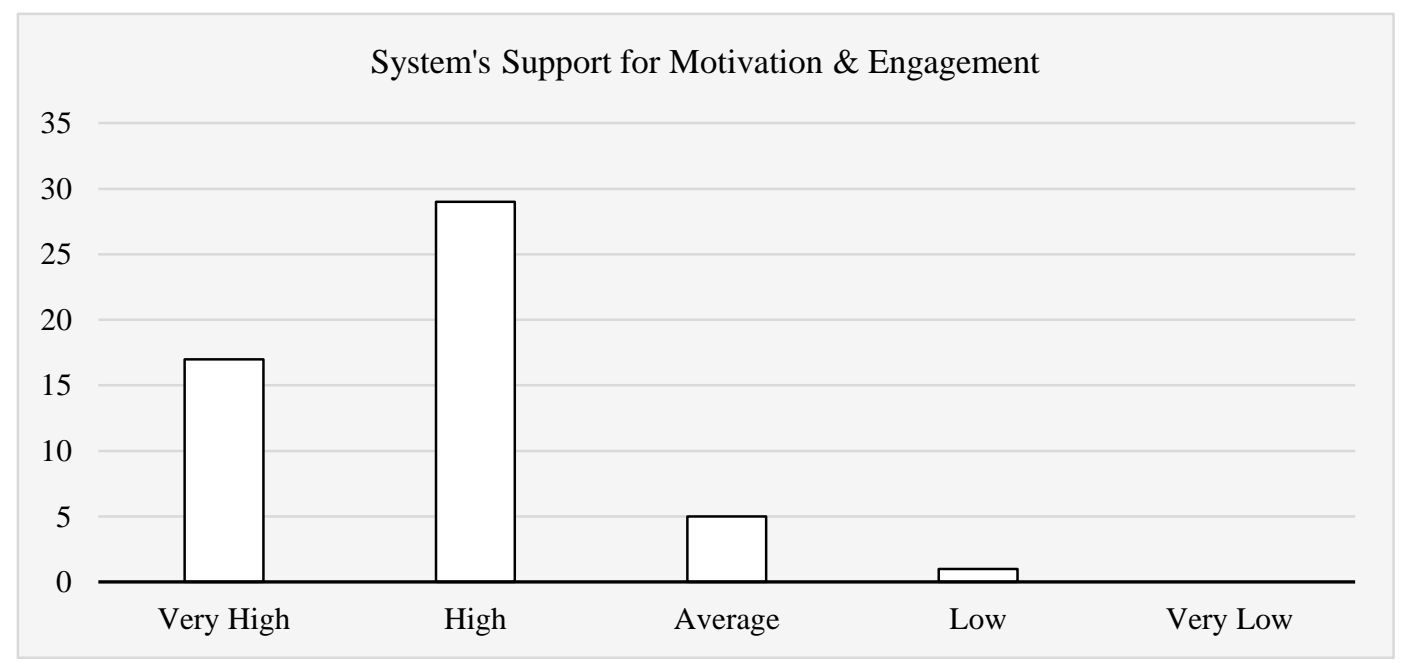

Figure 5: Participants’ Rating of System's Support for Motivation and Engagement 
There were 49 ratings in the high and very high levels with a single low and 5 average. Ratings in the haptics category were more distributed than for other categories as shown in Figure 6, with 33 overall high ratings, 12 average ratings and 7 in the low ratings category. This presents an interesting view, especially with the ability to support haptics being the focus of system development. Though data is still skewed in the direction of high ratings, participants' perception of the concept of haptics appear more diverse than for other elements. Individual interpretations or expectations of haptic perceptions might have contributed to this feedback. However, this objective is outside the scope of the present study and further investigation of individual's perception of the 'haptic' construct will be required to obtain more information on this.

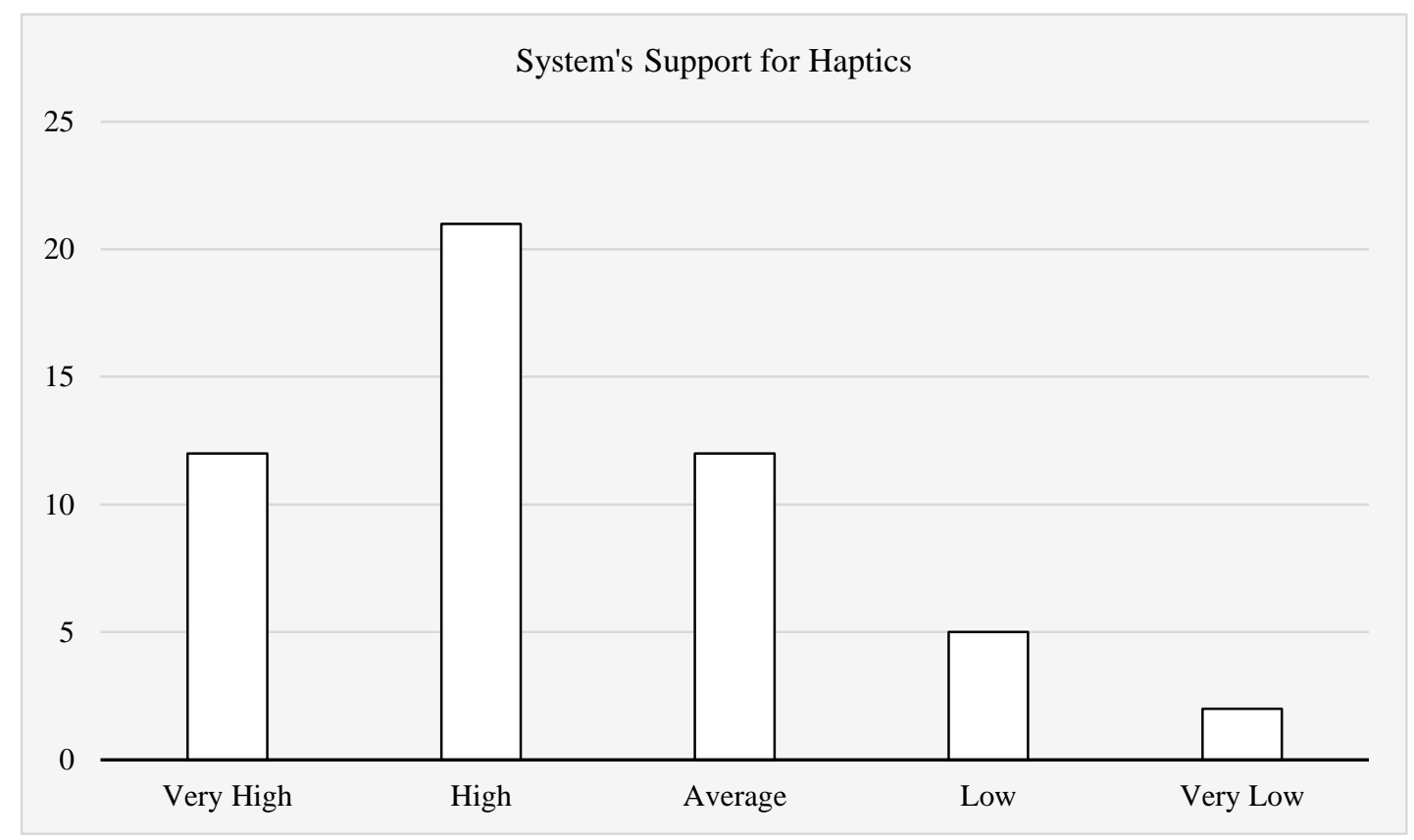

Figure 6: Participants’ Rating of System’s Support for Haptics

Figure 7 presents participants' perception of the adequacy of the system as an instructional tool. This is a general rating assessing system's ability to serve as educational technology or instructional material rather than specific use in a subject. Findings in this category were comparable to previous ones with no low ratings and only 2 average ratings. The remaining 50 are in the high categories.

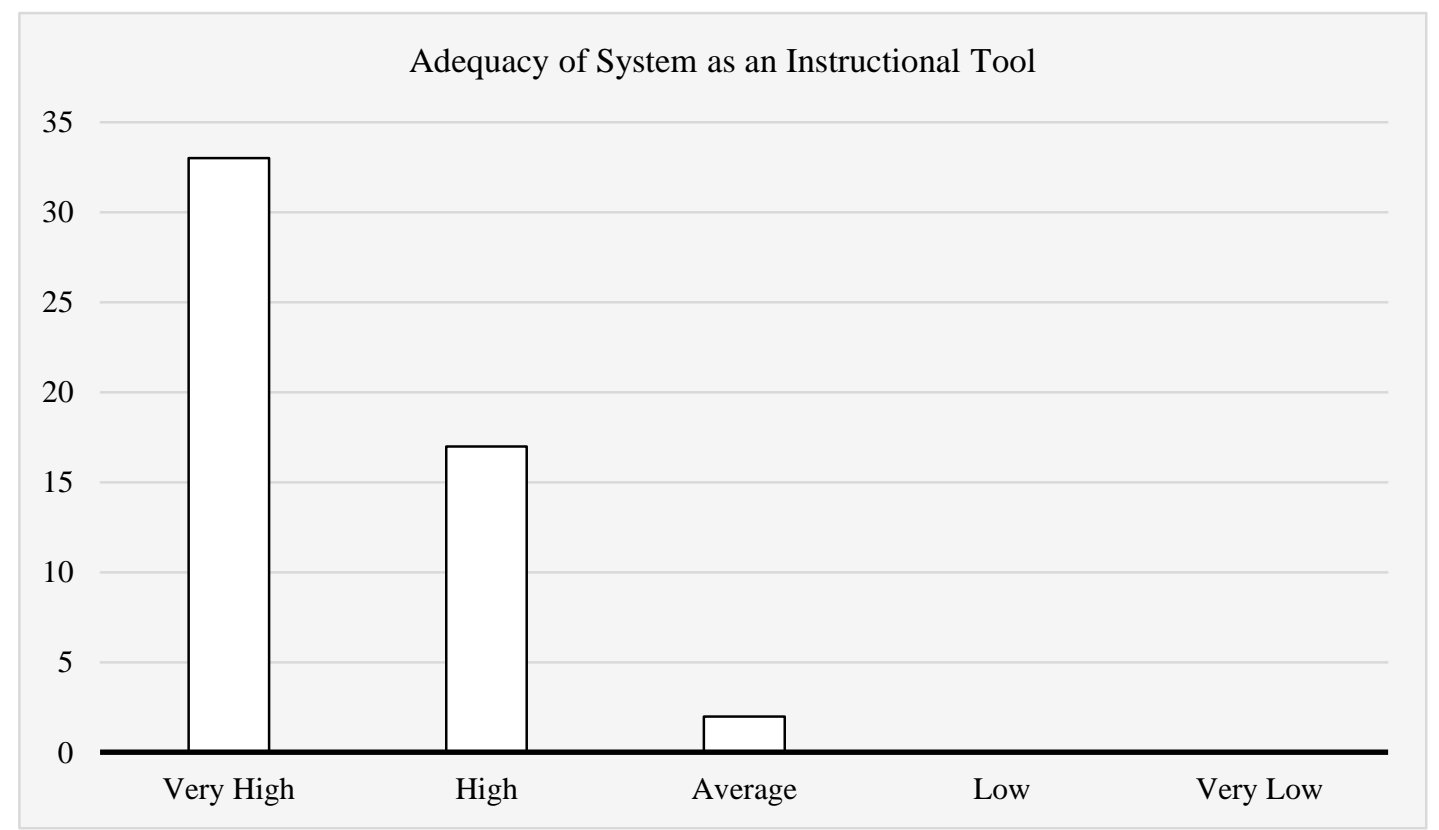

Figure 7: Participants’ Rating of Adequacy of System as an Instructional Tool 
Figure 8 presents ratings on suitability of system as a tool for chemistry instruction. The chart is similar to ratings of the system as a general instructional tool presented in Figure 7 (above). This feedback shows consistency in participants' feedback on the system and highlights the reliability of the feedback.

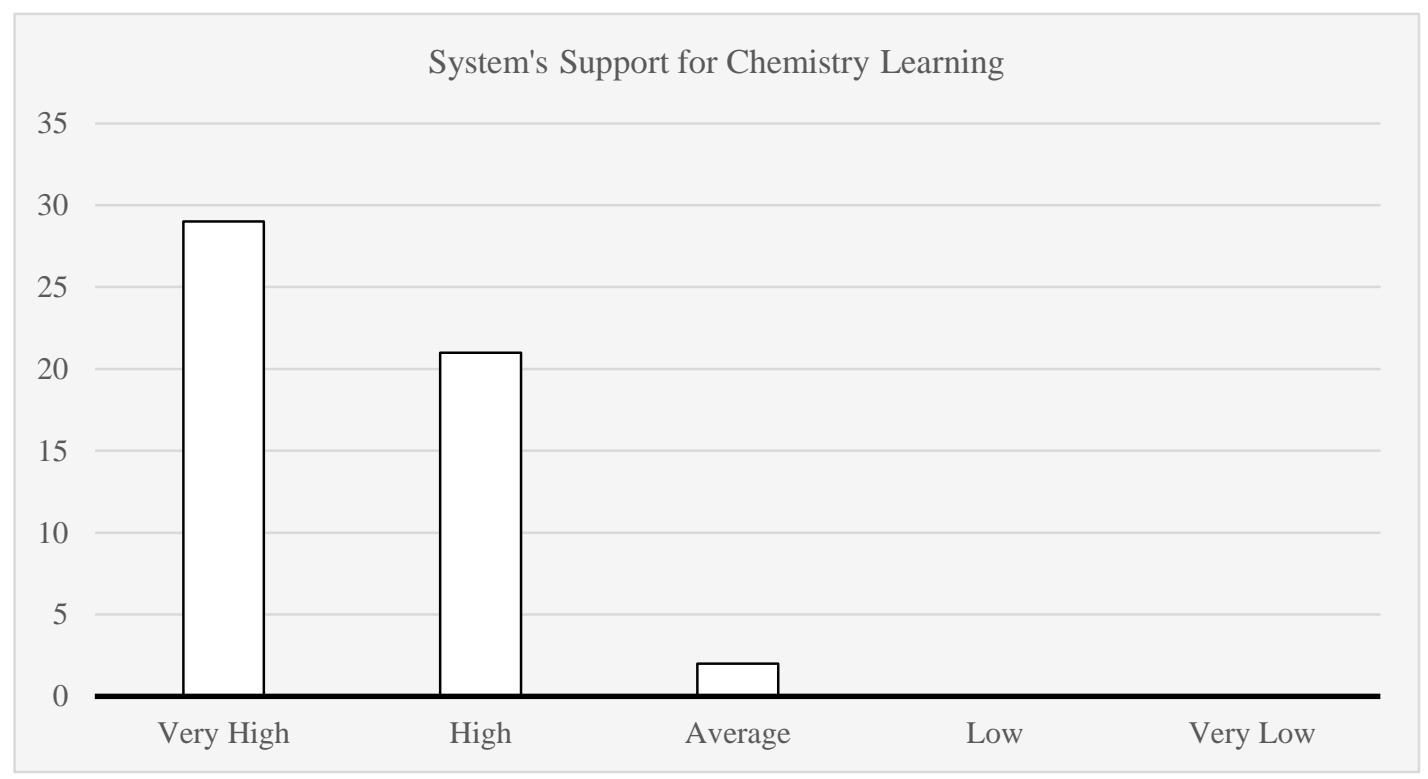

Figure 8: Participants’ Rating of Adequacy of System for Chemistry Instruction

In Figure 9, we present the overall rating of the system. Values are based on the sum of all feedbacks. Distribution of ratings are similar to ratings on the various sub-constructs presented above. There were mostly high ratings (37 of 52), no 'very low' and only 6 'low' ratings. The remaining 9 are in the average category. These findings indicate that, participants have very positive perception of the system, both based on various elements assessed as well as on its use as learning technology.

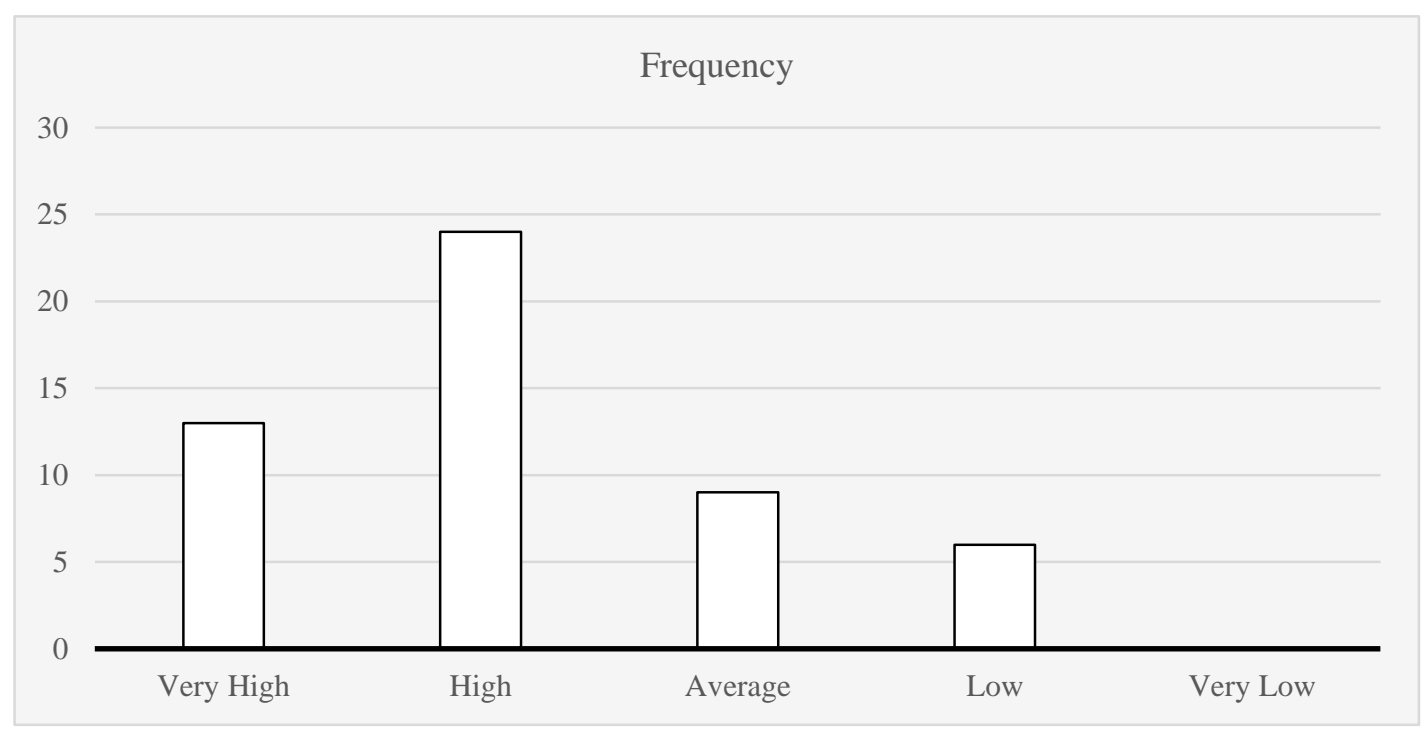

Figure 9: Participants’ Rating of Overall Perception of System

The statistical outputs with mean values and standard deviation are presented with the coefficient of variation (CV), in Table $1 . \mathrm{CV}$ is a ratio of SD to Mean, it provides a measure of relative variability in the data. Table 1 shows all CVs values are within 0.1 and 0.2 , that is, variance is low and values are closely dispersed around the mean of the data. 
Table 1: Descriptive Statistics for Participants' Evaluation of the VRMC

\begin{tabular}{|l|c|c|c|}
\hline Aspects of UUXQ & Mean & SD & $\begin{array}{c}\text { CV }= \\
\text { (SD/Mean) }\end{array}$ \\
\hline Multisensory learning & 17.15 & 1.91 & 0.11 \\
\hline Haptics & 14.77 & 3.03 & 0.21 \\
\hline Motivation and engagement & 16.77 & 3.4 & 0.20 \\
\hline Adequacy as a learning tool & 18.38 & 1.76 & 0.10 \\
\hline Adequacy for teaching chemistry & 18.08 & 2.02 & 0.11 \\
\hline
\end{tabular}

The values show that participants' responses were favourable for the indicators and the system was rated positively in terms of the purpose of its development.

\subsection{Open-ended Feedback}

All participants found the general design of the system impressive, interesting, and educative. They agreed that the VRMC has potential to serve as an instructional tool and particularly for teaching bonding and structure in organic chemistry. We will consider this in future system upgrades. Participants reported enjoyment and engagement and are confident that an improved version can support improved learning experience.

The need for more feedback to user (e.g. for identification of atoms and a built-in tutorial) and improved background graphics were noted. Feedback on the colour system was divergent; findings indicate user-preference as a strong factor and the possibility of user-setting for colour in future versions. Major improvement needs were noted regarding the haptic system and the gloves; the need for greater precision and sensitivity to support easier grasping were also reported. Participants also suggested the need for instruction provided as built-in audio (current version has no built-in instruction) with the possibility of a 'help' button. The only audio in the current prototype is the sound from the haptic feedback; participants suggested that the introductory tutorial should be deployed in audio format to support more immersive experience and user-engagement. Consideration for non-native English speakers was suggested by some users to improve usability.

Some of the limitations noted by users included the need for built-in audio for instruction and feedback, improved sensitivity and precision of the haptic system, and these items will be considered in the next version. We further noted that enabling repetition at user's request is also an important factor to be considered in future versions.

The wired glove connections make wearing the glove and reaching far out with the hands difficult. Almost all participants suggested a lighter, wireless glove system that supports users' free movement. Atom design was perceived very positively and noted as acceptable by all participants. There were suggestions that labelling the atoms can assist users who might not have a background in chemistry.

\subsection{Participant Observation}

User's experience of immersion was noted in their display of enjoyment and interest during observation. There were exclamations when they succeeded in 'picking' an atom. A participant was heard saying 'hey, come here!' while trying to pick up an atom; another said 'come on, don't go' addressing a 'falling' atom. A participant exclaimed, 'yeah! I made methane!' when she formed the methane molecule. Overall, live observation of users show that the system is practical and has the potential to promote improved engagement and motivation of users.

\section{DISCUSSION}

The current system is run from the Unity editor, using an Android phone connected with Unity Remote so that the PC can be used with the Leap Motion controller and the haptic gloves. This arrangement requires several wired connections from the headset and gloves to the computer, which imposes limitations on the user. We are planning for future versions to use wireless communication. Furthermore, the game could be modified to be a stand-alone application to eliminate wired connections and make it more suitable for use in a classroom setting, however, this would require different hand-tracking methods. Hand tracking in the current system is limited in 
volume, and it is responsible for 'dropped' atoms; this will be improved in future versions. The current system is designed for the basic chemistry of hydrocarbons but it can easily be modified to include other atoms just by creating additional virtual atomic models so that more complex organic molecules can be made to support advanced chemistry instruction. The chemical reactions involved in forming molecules can be simulated in future developments to give students a greater understanding of chemistry.

In comparison to existing systems, the VRMC demonstrates capabilities not currently available, that is, haptic VR for classroom instruction in basic organic chemistry. Findings from this evaluation highlight the effectiveness of the system as an educational technology and learning environment, highlighting how the immersive experience possible within VR can be augmented with haptics to promote game-like learning with an actual classroom subject. Users' perception of the system was high and responses in both quantitative and qualitative evaluations show that the VRMC has the potential to support teaching and learning of basic organic chemistry. System evaluation highlights the need for improved technology, wireless connections, and the use of in-built audio presentation of user instructions to further enhance the immersive experience.

Observation of user-experience with the system showed that written instructions were not effective with almost all participants; instructions had to be verbally repeated during trials. Because molecules built remain in the system, they sometimes obstruct visual navigation of the system; hence, we will consider the possibility of a 'molecule bank'. The system position drifts as user activity progresses, affecting user balance; in addition, initial calibration procedure was not clearly understood by users. We plan to consider more explicit instruction to assist users in manipulating atoms. Overall, qualitative feedback through observation is in line with participants' feedback from the quantitative results and in spite of the limitations of the current system, there are a lot of room for upgraded versions, and we are confident of significant improvement in future versions.

\section{CONCLUSIONS, IMPLICATIONS AND FUTURE STEPS}

The VRMC is a learning system that employs natural hand motion (haptics) for building molecules that use either or both hands for haptic experience in the learning of structure and bonding in organic chemistry in a VR environment. The system integrates the multiple advantages of immersive, multisensory, and tactile learning. According to Koedinger, et al. (2015), learning is not a 'spectator sport', hence, the current emphasis on the concept of 'learning by doing' which represent the basis for project- and scenario-based learning. This study is limited by the small size of respondents $(n=13)$, however, participants represent a diverse group of individuals, and the use of a mixed approach provides complementary and rich feedback to reduce the limitation of single designs using small samples. Qualitative and quantitative data on user experience support system usability to support multisensory learning, engagement, motivation, haptics and usefulness as learning technology.

Future versions will deploy different levels of gaming to extend the learning content to more topics. The VRMC demonstrates how VR technology is leveraged to provide an immersive learning experience that integrates several effective learning approaches including multimedia and multisensory instruction, haptics, and gamification of learning. The immersive VR environment supports simulation of abstract concepts like atoms and molecules, thereby promoting high engagement, motivation, and interest, which are known to promote enhanced memory (Turk et al., 2015). Within the immersive environment, the user is disengaged from physical distraction, the system can thus be useful for addressing the critical problem of learner engagement. In addition, the ability of users to view and touch atoms, molecules and bonds, supports the real-ness of abstracts as well as multisensory instruction. 


\section{REFERENCES}

Axon VR. (2017). Virtual Reality You Can Feel. Retrieved from https://axonvr.com/\#haptx-haptics-evolved Bazeley, P. (2010). Book Review: V. L. Plano Clark and J. W. Creswell (Eds.) The Mixed Methods Reader. Thousand Oaks, CA: SAGE, 2008, 617 pp. Supplied by Footprint Books, AU\$79 (paperback). Journal of Mixed Methods Research, 4(1), 79-81. https://doi.org/10.1177/1558689809356926

Buckley, P., \& Doyle, E. (2016). Gamification and student motivation. Interactive Learning Environments, 24(6), 1162-1175. https://doi.org/10.1080/10494820.2014.964263

Cambridge Dictionary. (2017). Definition of immersive. Cambridge Dictionary. Cambridge University Press. Retrieved from http://dictionary.cambridge.org/dictionary/english/immersive

Choi, S., Jung, K., \& Noh, S. D. (2015). Virtual reality applications in manufacturing industries: Past research, present findings, and future directions. Concurrent Engineering Research and Applications, 23(1), 40-63. https://doi.org/10.1177/1063293X14568814

Christou, C. (2010). Virtual Reality in Education. Education, (November), 228-230. https://doi.org/10.4018/978-1-60566-940-3.ch012

Creswell, J. W. (2014). Research Design: Qualitative, Quantitative and Mixed Approaches. Research Design: Qualitative, Quantitative, and Mixed Methods Approaches (4th ed.). https://doi.org/10.2307/1523157

Creswell, J. W., \& Plano Clark, V. L. (2007). Choosing a Mixed Method Design. In Designing and Conducting Mixed Methods Research (pp. 58-89). SAGE Publications, Inc. https://doi.org/1412927927

Culatta, R. (2013). ADDIE Model.

DextaRobotics. (2018). DextaRobotics Builds Hand Haptics Device for Virtual Reality Medical Learning. Retrieved March 21, 2018, from https://www.healthysimulation.com/9009/dextarobotics-builds-handhaptics-device-for-virtual-reality-medical-learning/

Dünser, A., Steinbügl, K., Kaufmann, H., \& Glück, J. (2006). Virtual and augmented reality as spatial ability training tools. In Proceedings of the 6th ACM SIGCHI New Zealand chapter's international conference on Computer-human interaction design centered HCI - CHINZ '06 (Vol. 158, pp. 125-132). https://doi.org/10.1145/1152760.1152776

Eastwood, M. L. (2013). Fastest fingers: A molecule-building game for teaching organic chemistry. Journal of Chemical Education, 90(8), 1038-1041. https://doi.org/10.1021/ed3004462

Erlandson, D. A., Harris, E. L., Skipper, B. L., \& Allen, S. D. (1993). Doing Naturalistic Inquiry: A Guide to Methods. SAGE Publications, Inc.

Farra, S. L., Miller, E. T., \& Hodgson, E. (2015). Virtual reality disaster training: Translation to practice. Nurse Education in Practice, 15(1), 53-57. https://doi.org/10.1016/j.nepr.2013.08.017

Fildes, N. (2015, December). 2016 set to be year virtual reality takes off. Raconteur. Retrieved from https://www.raconteur.net/technology/2016-set-to-be-year-virtual-reality-takes-off

Google. (2017). Virtual reality for everyone. Retrieved November 9, 2017, from https://vr.google.com/

GoTouch VR Team. (2017). Go Touch VR. Retrieved November 9, 2017, from https://www.gotouchvr.com/

Hamid, N. S. S., Aziz, F. A., \& Azizi, A. (2014). Virtual reality applications in manufacturing system. Proceedings of 2014 Science and Information Conference, SAI 2014, 1034-1037. https://doi.org/10.1109/SAI.2014.6918317

Hauptman, H. (2010). Enhancement of spatial thinking with Virtual Spaces 1.0. Computers and Education, 54(1), 123-135. https://doi.org/10.1016/j.compedu.2009.07.013

Heinich, R., Molenda, M., Russell, J., \& Smaldino, S. (2002). The ASSURE Model. In Instructional Media and Technologies for Learning (Vol. Seventh).

Hyun, E. H. E., Yoon, H. Y. H., \& Son, S. S. S. (2010). Relationships between user experiences and children's perceptions of the education robot. Human-Robot Interaction (HRI), 2010 th ACM/IEEE International Conference on, 199-200. https://doi.org/10.1109/HRI.2010.5453197

Jong, T. (2009). Cognitive load theory, educational research, and instructional design: some food for thought. Instructional Science, 38(2), 105-134. https://doi.org/10.1007/s11251-009-9110-0

Koedinger, K. R., Kim, J., Jia, J. Z., McLaughlin, E. A., \& Bier, N. L. (2015). Learning is not a spectator sport: doing is better than watching for learning from a MOOC. Proceedings of the Second (2015) ACM Conference on Learning @ Scale - L@S '15, 111-120. https://doi.org/10.1145/2724660.2724681

Kolb, A. Y., \& Kolb, D. A. (2005). The Kolb Learning Style Inventory — Version 3.12005 Technical Specifications. LSI Technical Manual, 1-72. https://doi.org/10.1016/S0260-6917(95)80103-0

Kolb, D. A., Boyatzis, Richard, E., \& Mainemelis, C. (2000). Experiential Learning Theory: Previous Research and New Directions. Perspectives on Thinking Learning and Cognitive Styles, 1(216), 227-247. https://doi.org/10.5465/AMLE.2005.17268566

Kuo, M. S., \& Chuang, T. Y. (2016). How gamification motivates visits and engagement for online academic dissemination - An empirical study. Computers in Human Behavior, 55, 16-27. https://doi.org/10.1016/j.chb.2015.08.025

Leap Motion. (2017). Reach into Virtual Reality with your bare hands. Retrieved from https://www.leapmotion.com/\#112 
Mei, H. H., \& Sheng, L. S. (2011). Applying Situated Learning in a Virtual Reality System to Enhance Learning Motivation. International Journal of Information and Education Technology, 1(4), 298-302. https://doi.org/10.7763/IJIET.2011.V1.48

MEL Chemistry. (2017). MEL Chemistry VR. Retrieved from https://melscience.com/vr/

Merchant, Z., Goetz, E. T., Keeney-Kennicutt, W., Cifuentes, L., Kwok, O., \& Davis, T. J. (2013). Exploring 3 $\mathrm{D}$ virtual reality technology for spatial ability and chemistry achievement. Journal of Computer Assisted Learning, 29(6), 579-590. https://doi.org/10.1111/jcal.12018

Merriam Webster Dictionary. (2017). Definition of immersive.

Mestre, D., \& Vercher, J.-L. (2011). Immersion and Presence. In Virtual Reality: Concepts and Technologies (pp. 81-96). Retrieved from http://www.ism.univmed.fr/mestre/projects/virtual reality/Pres_2005.pdf

Minogue, J., \& Jones, M. G. (2006). Haptics in Education: Exploring an Untapped Sensory Modality. Review of Educational Research, 76(3), 317-348. https://doi.org/10.3102/00346543076003317

Molenda, M. (2003a). ADDIE Model. Retrieved from http://www.nwlink.com/ donclark/history_isd/addie.html\#FSU

Molenda, M. (2003b). ADDIE Model.

Monahan, T., McArdle, G., \& Bertolotto, M. (2008). Virtual reality for collaborative e-learning. Computers and Education, 50(4), 1339-1353. https://doi.org/10.1016/j.compedu.2006.12.008

Mujber, T., Szecsi, T., \& Hashmi, M. (2004). Virtual reality applications in manufacturing process simulation. Journal of Materials Processing Technology, 155156, 1834-1838. https://doi.org/10.1016/j.jmatprotec.2004.04.401

Nakamura, J., \& Csikszentmihalyi, M. (2009). Flow theory and research. Oxford Handbook of Positive Psychology. https://doi.org/10.1093/oxfordhb/9780195187243.013.0018

Norrby, M., Grebner, C., Eriksson, J., \& Bostrom, J. (2015). Molecular Rift: Virtual Reality for Drug Designers. Journal of Chemical Information and Modeling, 55(11), 2475-2484. https://doi.org/10.1021/acs.jcim.5b00544

Ornstein, A. (2006). The frequency of hands-on experimentation and student attitudes toward science: A statistically significant relation (2005-51-ornstein). Journal of Science Education and Technology, 15(34), 285-297. https://doi.org/10.1007/s10956-006-9015-5

React! Team. (2017). React! The Organic Chemistry Board Game. Retrieved June 22, 2017, from http://reactgame.com/\#/

Ritter, D., \& Johnson, M. (1997). Virtual Titrator: A Student-Oriented Instrument. Journal of Chemical Education, 74(1), 120. https://doi.org/10.1021/ed074p120

Sanders, T., \& Cairns, P. (2010). Time perception, immersion and music in videogames. Proceedings of the 24th BCS Interaction Specialist Group Conference, 160-167.

Schell Games. (2017). SuperChem VR. Retrieved June 20, 2017, from https://www.schellgames.com/games/superchem-vr

Smith, C., Agcaoili, K., \& Kannan, A. (2016). Chemistry Lab VR. Retrieved May 20, 2017, from https://devpost.com/software/chemistry-lab-vr

Stohr-Hunt, P. M. (1996). An analysis of frequency of hands-on experience and science achievement. Journal of Research in Science Teaching, 33(1), 101-109. https://doi.org/10.1002/(SICI)10982736(199601)33:1<101::AID-TEA6>3.0.CO;2-Z

Stone, D. C. (2007). Teaching Chromatography Using Virtual Laboratory Exercises. Journal of Chemical Education, 84(9), 1488-1495. https://doi.org/10.1021/ed084p1488

Turk, D. J., Gillespie-Smith, K., Krigolson, O. E., Havard, C., Conway, M. A., \& Cunningham, S. J. (2015). Selfish learning: The impact of self-referential encoding on children's literacy attainment. Learning and Instruction, 40, 54-60. https://doi.org/10.1016/j.learninstruc.2015.08.001

Tuveri, E., Macis, L., Sorrentino, F., Spano, L. D., \& Scateni, R. (2016). Fitmersive Games : Fitness Gamification through Immersive VR. In Proceedings of the International Working Conference on Advanced Visual Interfaces - AVI '16 (pp. 212-215). https://doi.org/10.1145/2909132.2909287

Unimersiv. (2016). Chemistry VR. Retrieved June 20, 2017, from https://unimersiv.com/review/chemistry-vr/

Whitson, C., \& Consoli, J. (2009). Flow Theory and Student Engagement. Journal of Cross-Disciplinary Perspectives in Education, 2(1), 40-49. Retrieved from http://jcpe.wmwikis.net/file/view/whitsonconsoli.pdf

Winkelmann, K., Scott, M., \& Wong, D. (2014). A study of high school students' performance of a chemistry experiment within the virtual world of second life. Journal of Chemical Education, 91(9), 1432-1438. https://doi.org/10.1021/ed500009e

Winter, J., Wentzel, M., \& Ahluwalia, S. (2016). Chairs!: A Mobile Game for Organic Chemistry Students to Learn the Ring Flip of Cyclohexane. Journal of Chemical Education, 93(9), 1657-1659. https://doi.org/10.1021/acs.jchemed.5b00872

Woolley, J., Sheeley, J., \& Sheeley, S. (2010). Organic Molecule Game. Retrieved from http://chem.illinois.edu/omg/index.html

Yiannakopoulou, E., Nikiteas, N., Perrea, D., \& Tsigris, C. (2015). Virtual reality simulators and training in 
laparoscopic surgery. International Journal of Surgery. https://doi.org/10.1016/j.ijsu.2014.11.014 